\title{
LESÕES TRAUMÁTICAS DE VÍSCERAS OCAS
}

\author{
INJURIES TO THE HOLLOW VISCERA
}

Dino Cesar Motta ${ }^{1}$, Sandro Scarpelini²

${ }^{1}$ Médico Assistente. ${ }^{2}$ Docente. Disciplina de Cirurgia de Urgência e Trauma. Departamento de Cirurgia e Anatomia. Faculdade de Medicina de Ribeirão - USP.

CorRespondência: Centro de Estudos de Emergências em Saúde. Rua Bernardino de Campos, $1000,2^{\circ}$ andar

14015-130 Ribeirão Preto - SP. e-mail: sandro@fmrp.usp.br

Motta DC, Scarpelini S. Lesões traumáticas de vísceras ocas. Medicina (Ribeirão Preto) 2007; 40 (4): 531-7, out./dez.

RESUMO: Nos pacientes vítimas de traumatismo abdominal, contuso ou penetrante, além dos cuidados iniciais de estabilização, diagnóstico cuidadoso de lesões associadas e tratamento cirúrgico imediato em situações óbvias, devemos estar atentos para possíveis lesões de vísceras ocas que podem se manifestar tardiamente, postergando o tratamento definitivo, com conseqüente piora do prognóstico. Baseado na forte suspeita, analisando o mecanismo do trauma e lesões encontradas na avaliação inicial, o lavado peritoneal diagnóstico e exames de imagem devem ser empregados em conjunto, para confirmar ou afastar a existência de lesão de vísceras ocas. Atualmente, não dispomos de exames com especificidade e sensibilidade suficientes para, isoladamente, definir o diagnóstico. Dentre os órgãos intra cavitários não sólidos envolvidos nos traumatismos abdominais, destaca-se o intestino delgado como o mais acometido nas lesões penetrantes, seguido pelo cólon, estômago e duodeno. As lesões de vísceras ocas são menos freqüentes em traumatismos contusos, porém, geralmente, apresentam-se com maior gravidade e associadas a outras lesões. O tratamento é cirúrgico, em geral com o reparo primário da víscera, sendo a tática operatória guiada pela gravidade das lesões e condições gerais do paciente.

Descritores: Traumatismos Abdominais. Vísceras; lesões. Estômago; lesões. Duodeno; lesões. Intestino Delgado; lesões. Cólon; lesões.

\section{1- INTRODUÇÃO}

Dentre as lesões encontradas nos pacientes vítimas de traumatismo, destacamos as que acometem vísceras ocas devido a sua alta morbidade e mortalidade, especialmente se o diagnóstico for postergado. Embora o mecanismo de trauma permita inferências sobre o tipo de lesão, o diagnóstico é notoriamente dificultado, tanto pela falta de achados clínicos e radiológicos específicos, quanto por estar associado a outras lesões graves que mascaram ou impedem uma avaliação adequada do abdômen. Pacientes com comprometimento do nível de consciência são exemplos típicos desta situação.

As lesões de vísceras ocas produzem sintomas pela combinação de perda sanguínea e contaminação peritoneal, podendo, portanto, demorar várias horas para se manifestar clinicamente. Outro fator complicador para o diagnóstico é a existência de porções retroperitoneais do tubo digestivo, que podem levar a interpretações inadequadas de exames utilizados na propedêutica do trauma.

O trauma da parede anterior do abdômen, contuso ou penetrante, frequientemente leva a lesões de vísceras ocas, embora ferimentos de dorso e nádegas possam também atingir órgãos intra-cavitários. A gravidade das lesões é graduada segundo os índices elaborados pela "American Association for the Surgery of Trauma" (AAST), também chamados de "Organ Injury Scale" (OIS) (Quadro I), e a gravidade da lesão, em geral, norteia o tratamento definitivo. ${ }^{1}$ 


\begin{tabular}{|c|c|c|}
\hline Órgão lesado & Grau & Características da lesão \\
\hline \multirow{5}{*}{ ESTÔMAGO } & I & $\begin{array}{l}\text { Hematoma: contusão ou hematoma sem desvascularização; } \\
\text { Laceração: lesão superficial sem perfuração. }\end{array}$ \\
\hline & II & $\begin{aligned} \text { Laceração: } & <2 \mathrm{~cm} \text { na junção gastroesofágica ou piloro; } \\
& <5 \mathrm{~cm} \text { no } 1 / 3 \text { proximal do estômago; } \\
& <10 \mathrm{~cm} \text { no } 2 / 3 \text { distal do estômago. }\end{aligned}$ \\
\hline & III & $\begin{aligned} \text { Laceração: } & >2 \mathrm{~cm} \text { na junção gastroesofágica ou piloro; } \\
& \geq 5 \mathrm{~cm} 1 / 3 \text { proximal do estômago; } \\
& \geq 10 \mathrm{~cm} \text { no } 2 / 3 \text { distal do estômago. }\end{aligned}$ \\
\hline & $\mathrm{IV}$ & Laceração: perda de tecido ou desvascularização $\leq 2 / 3$ do estômago. \\
\hline & V & Laceração: perda de tecido ou desvascularização $\geq 2 / 3$ do estômago. \\
\hline
\end{tabular}

\begin{tabular}{|c|c|c|}
\hline \multirow{5}{*}{ DUODENO } & I & $\begin{array}{l}\text { Hematoma: contusão envolvendo uma única porção; } \\
\text { Laceração: lesão superficial sem perfuração. }\end{array}$ \\
\hline & II & $\begin{array}{l}\text { Hematoma: contusão envolvendo mais de uma porção; } \\
\text { Laceração: laceração < } 50 \% \text { da circunferência. }\end{array}$ \\
\hline & III & 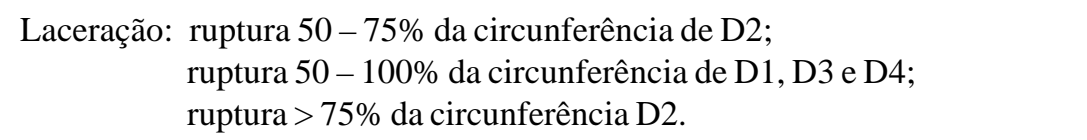 \\
\hline & IV & Laceração: envolvendo ampola ou ducto biliar comum. \\
\hline & $\mathrm{V}$ & $\begin{array}{l}\text { Laceração: destruição maciça do complexo duodenopancreático; } \\
\text { desvascularização do duodeno. }\end{array}$ \\
\hline \multirow{5}{*}{$\begin{array}{l}\text { INTESTINO } \\
\text { DELGADO }\end{array}$} & I & $\begin{array}{l}\text { Hematoma: contusão ou hematoma sem desvascularização; } \\
\text { Laceração: lesão superficial sem perfuração. }\end{array}$ \\
\hline & II & Laceração: laceração < 50\% da circunferência. \\
\hline & III & Laceração: laceração $\geq 50 \%$ da circunferência sem transecção. \\
\hline & IV & Laceração: transecção do intestino delgado com perda segmentar de tecido. \\
\hline & $\mathrm{V}$ & Vascular: desvascularização segmentar. \\
\hline \multirow{5}{*}{ CÓLON } & I & $\begin{array}{l}\text { Hematoma: contusão ou hematoma sem desvascularização; } \\
\text { Laceração: lesão superficial sem perfuração. }\end{array}$ \\
\hline & II & Laceração: laceração $<50 \%$ da circunferência. \\
\hline & III & Laceração: laceração $\geq 50 \%$ da circunferência sem transecção. \\
\hline & $\mathrm{IV}$ & Laceração: transecção do cólon. \\
\hline & $\mathrm{V}$ & Laceração: transecção do cólon com perda segmentar de tecido. \\
\hline \multirow{5}{*}{ RETO } & I & $\begin{array}{l}\text { Hematoma: contusão ou hematoma sem desvascularização; } \\
\text { Laceração: lesão superficial sem perfuração. }\end{array}$ \\
\hline & II & Laceração: laceração < 50\% da circunferência. \\
\hline & III & Laceração: laceração $\geq 50 \%$ da circunferência sem transecção. \\
\hline & IV & Laceração: laceração profunda com extensão para o períneo. \\
\hline & $\mathrm{V}$ & Vascular: devascularização segmentar. \\
\hline
\end{tabular}


A lesão de víscera oca é mais comum no trauma abdominal penetrante do que no trauma fechado, atingindo os seguintes órgãos, pela ordem de frequiência: intestino delgado, cólon, estômago e duodeno. ${ }^{2}$ Nesta revisão, as lesões desses órgãos e o seu tratamento definitivo serão discutidos individualmente, devido a suas peculiaridades.

Salientamos que, frente a qualquer paciente vítima de trauma, as primeiras condutas são sempre voltadas para a estabilização fisiológica, seguindo a sequiência preconizada pelo curso "Advanced Trauma Life Suport" (ATLS ${ }^{\circledR}$ ), com a priorização, identificação e tratamento das lesões que põem em risco imediato a vida do paciente. ${ }^{3}$ Do ponto de vista cirúrgico, nos útlimos anos, tem sido desenvolvido o conceito de cirurgia para o controle de danos ("damage control"), ou laparotomia abreviada, com objetivo de controlar rapidamente o sangramente maciço e possíveis fontes de infecção, levando o paciente, imediatamente, para o ambiente de terapia intensiva para reestabelecimento da homeostasia. ${ }^{4}$ Em um segundo tempo, o paciente é submetido à nova laparotomia, na tentativa de se obter o tratamento definitivo das lesões. Do ponto de vista de vísceras ocas, de maneira geral, esta abordagem significa o fechamento provisório das lesões (fechamento de cotos cegos, grampeamento provisório de feridas e empacotamento com compressas cirúrgicas), para proceder às reconstruções definitivas quando o paciente estiver em melhores condições gerais. ${ }^{5}$

O exame físico continua a ser o mais importante instrumento para diagnosticar lesões abdominais que necessitem de laparotomia de urgência. Métodos diagnósticos auxiliares nos traumas abdominais incluem o Lavado Peritoneal Diagnóstico (LPD), exame ultrasonográfico "Focused Assessment with Sonography for Trauma" - FAST ${ }^{3}$ e a Tomografia Computadorizada (TC), cuja evolução tecnológica tem permitindo a identificação de lesões sutis. ${ }^{6} \mathrm{O}$ emprego de procedimentos laparoscópicos nas emergências não traumáticas tem sido estabelecido, entretanto, com relação ao seu uso no trauma (contuso ou penetrante), as opiniões são controversas, ainda necessitando de mais estudos para comprovação de sua eficiência. ${ }^{7}$

\section{2- LESÃO TRAUMÁTICA DO ESTÔMAGO}

Lesões gástricas no trauma são, na maioria das vezes, associadas a traumatismo penetrante, sendo raras nos traumas fechados com incidência de 0,02 a
$1,7 \% .{ }^{8} \mathrm{~A}$ incidência em crianças pode ser mais elevada, possivelmente pela maior elasticidade da parede anterior do abdome nesta faixa etária. Quando presente em traumatismos fechados está associado a outras lesões, extra ou intra-abdominais, sendo a lesão esplênica a associação mais comum. Os ferimentos geralmente se situam na parede anterior e na grande curvatura gástrica, produzindo extravasamento de conteúdo e contaminação da cavidade peritoneal. A alta morbidade e mortalidade estão diretamente relacionadas às lesões associadas, demora no diagnóstico e desenvolvimento de sepse abdominal. As lesões gástricas isoladas são raras, porém associadas a menor morbimortalidade. ${ }^{9}$

O diagnóstico das lesões viscerais nos traumas penetrantes é, na maioria das vezes, feito na laparotomia, uma vez que a indicação da cirurgia é decorrente da violação da cavidade peritoneal. A presença de sangue na sonda gástrica e o achado de pneumoperitôneo no raio $\mathrm{X}$ podem ser sinais de lesões gástricas, porém, nenhum sinal é patognomônico dessas lesões. Dentre os métodos diagnósticos adjuntos ao exame físico, o LPD é o mais sensível (98\%), porém pouco específico para identificar a lesão do estômago ou qualquer outra órgão lesado. ${ }^{3}$

A tática cirúrgica para correção definitiva dos ferimentos gástricos depende da gravidade da lesão e da condição geral do paciente. Do ponto de vista anatômico, as condutas podem ser classificadas de acordo com a escala OIS ${ }^{2}$ :

\section{Lesões grau I, II ou III}

Parcial: hemostasia e sutura seromuscular

Total: hemostasia e sutura em dois planos

\section{Lesões grau IV - (Não sujeita a reparo primário)}

- Sem lesões associadas de duodeno, pâncreas ou esôfago:

- Gastrectomia distal com reconstrução à Bilroth I

- Com lesões associadas de duodeno ou pâncreas:

- Gastrectomia distal com reconstrução à Bilroth II

- Gastrectomia total com reconstrução à Y de Roux

\section{3- LESÃO TRAUMÁTICA DE INTESTINO DELGADO}

O intestino delgado é a víscera mais atingida nos traumatismos abdominais penetrantes devido ao grande volume que ocupa no abdômen. A lesão de delgado é pouco freqüente nas contusões, embora 
sejam encontradas nos traumas com grande energia cinética como acidentes automobilísticos em alta velocidade. Usualmente, são decorrentes da desaceleração que produz um estiramento dos pontos de fixação visceral, como angulo de Treitz e da região ileocecal. Também são relacionadas com uma compressão abrupta e localizada, pelo uso de cinto de segurança. Em pacientes traumatizados que se apresentam com equimoses abdominais lineares (sinal do cinto de segurança) a possibilidade de lesão de intestino delgado deve ser aventada e esforços para seu diagnóstico precoce não podem ser poupados. ${ }^{3}$

Geralmente as lesões de delgado no trauma fechado são isoladas podendo, todavia, estar associadas à fratura transversal de coluna lombar (Fratura de Chance), a qual também é relacionada com o uso de cinto de segurança. ${ }^{3}$ A lesão de mesentério agrava o quadro devido a possível hemorragia grave e isquemia segmentar.

Frente à escassez de sinais clínicos e radiológicos específicos e confiáveis na maioria dos casos, o diagnóstico de lesões intestinais é um desafio para o cirurgião e para o radiologista. ${ }^{10} \mathrm{O}$ mecanismo de trauma e o alto grau de suspeita são o melhor guia para o diagnóstico das lesões. Exames complementares devem ser usados, porém o valor preditivo do FAST em casos de lesão de intestino delgado é de apenas $38 \%$, e a sensibilidade e especificidade da TC não chega a $80 \% .{ }^{11}$ A presença de líquido livre na cavidade na ausência de lesão de víscera parenquimatosa e o espessamento da parede intestinal, como únicos achados, ou a presença de pneumoperitôneo podem identificar a lesão de víscera oca. ${ }^{6,11}$ O LPD pode ser mais sensível, embora seja passível de complicações como lesões de vísceras e resultado falso positivo ou negativo. $^{3}$

Em resumo, o sucesso no diagnóstico de lesões de intestino delgado não evidentes requer uma ampla avaliação levando em conta os achados clínicos e a história do trauma, além de exames complementares seriados e um período prolongado de observação. ${ }^{11}$

O tratamento definitivo das lesões traumáticas do intestino delgado é cirúrgico, variando a técnica empregada de acordo com o grau de gravidade da lesão. ${ }^{11}$

Grau I: hemostasia e sutura sero-muscular;

Grau II: debridamento e fechamento primário em 1 ou 2 planos;

Grau II (grande) ou Grau III a V: ressecção segmentar e anastomose primária. ${ }^{2}$

\section{4- LESÃO TRAUMÁTICA DE DUODENO}

As lesões duodenais são incomuns, porém graves e respondem por uma alta morbidade e mortalidade. Diferenças entre os mecanismos de trauma, gravidade das lesões associadas e o tempo entre o trauma, o diagnóstico e o tratamento influenciam na evolução do caso. Na maioria das vezes é resultado de lesão penetrante. Em $20 \%$ dos casos ocorrem lesões associadas com o pâncreas, o que piora sobremaneira o prognóstico. ${ }^{2}$ A alta taxa de complicações associadas a este tipo de lesão se deve à demora no diagnóstico e/ou lesões despercebidas, decorrente da natureza insidiosa de algumas lesões duodenais, além de sua localização anatômica retroperitoneal.

Os sinais radiológicos nas lesões duodenais são sutis e indiretos. Uma forte suspeita deve ser confirmada ou excluída pela realização de TC abdominal associada a seriografia com contraste hidrossolúvel, nos pacientes hemodinamicamente estáveis. Alterações nesses exames, mesmo que discretas, devem indicar laparotomia uma vez que a abordagem e a evolução de lesões tardias são catastróficas. O LPD não é confiável para detectar lesões isoladas de duodeno ou outras vísceras retroperitoneais. ${ }^{2}$

O tratamento do trauma duodenal é determinado pela probabilidade de complicações e gravidade da lesão. A gravidade das lesões pode ser classificada pela escala da AAST (Quadro I). As lesões de grau I e II respondem por 70 a $80 \%$ dos ferimentos duodenais e são passíveis de reparo primário. ${ }^{2}$ As lesões de grau III a V são menos freqüentes e requerem procedimentos cirúrgicos mais complexos para seu tratamento definitivo, incluindo exclusão pilórica, anastomose duodeno-jejunal, duodenoduodenoanastomose ou mais raramente duodenopancreatectomia, realizada apenas em casos de lesões com hemorragia incontrolável de pâncreas ou lesão associada de ductos biliares ou pancreáticos. $^{2}$

Mortes precoces nos ferimentos duodenais, em geral, são atribuídas à lesões associadas de grandes vasos, do fígado ou baço. A localização anatômica do duodeno torna incomuns as lesões isoladas deste órgão. Mortes mais tardias são associadas à infecção e falência de múltiplos órgãos. Em geral, a abordagem cirúrgica do trauma duodenal objetiva o controle da hemorragia, seguido do cuidado em limitar a contaminação proveniente do extravasamento do conteúdo digestivo e a identificação das lesões pancreáticas e de vias biliares. 


\section{5- LESÃO TRAUMÁTICA DE CÓLON E RETO}

O cólon figura como o segundo órgão mais acometido em lesões traumáticas penetrantes, logo após o intestino delgado. ${ }^{2}$ As lesões decorrentes de trauma contuso são mais raras, em torno de 2 a $5 \%$ dos casos, porém são mais extensas e mais graves. Geralmente estão associadas a outras lesões, as quais dificultam o diagnóstico e pioram o prognóstico. ${ }^{12}$ Outro fator para o aumento da morbidade é a demora no diagnóstico, portanto, um alto índice de suspeita é necessário mesmo na ausência de sinais clínicos no início da avaliação. Além do exame físico acurado, o diagnóstico é excluído ou confirmado por uma combinação de métodos, incluindo LPD, FAST e TC, como para as demais vísceras ocas, uma vez que nenhum método atual, isoladamente, é suficiente para concluir o diagnóstico. ${ }^{13} \mathrm{O}$ emprego de laparoscopia pode ser útil sob alguns aspectos, porém, como já foi discutido, a indicação precisa para seu uso ainda não está completamente estabelecida. ${ }^{13}$

A correta abordagem nas lesões de cólon vem sendo debatida e tem evoluído ao longo das últimas décadas. A maior controvérsia diz respeito ao reparo primário da lesão ou o emprego de derivações intesti- nais. Estudos controlados, baseados em evidências, estabeleceram a segurança do reparo primário nas lesões colônicas, mas salientam que a experiência do cirurgião é fundamental para avaliar a boa irrigação e a ausência de tensão e edema dos segmentos a serem tratados primariamente. ${ }^{14,15} \mathrm{O}$ tratamento cirúrgico das lesões traumáticas de colón é sumarizado no algoritmo da Figura 1.

Nos pacientes com ferimentos de períneo, pélvis e nádegas deve se supor a possível lesão de reto. O exame digital, com toque retal, é mandatório à procura de sangramento e espículas ósseas, embora a ausência destes não exclua o diagnóstico de lesão retal. Está indicada a realização de retossigmoidoscopia sempre que houver suspeita de ferimento de reto. Lesões associadas de bexiga, uretra e de vasos pélvicos são freqüentes.

O tratamento cirúrgico das lesões de reto alto intraperitoneal é semelhante ao das lesões colônicas (Figura 1). Lesões de reto extraperitoneais são tratadas por sutura (se acessível) e a necessidade de colostomia protetora deve se avaliada. Apesar de controverso o emprego de drenagem pré sacral pode ser aventada associada à colostomia protetora em casos de lesões não alcançáveis. ${ }^{13,16}$ De maneira geral, as drenagens não são indicadas pelos autores.

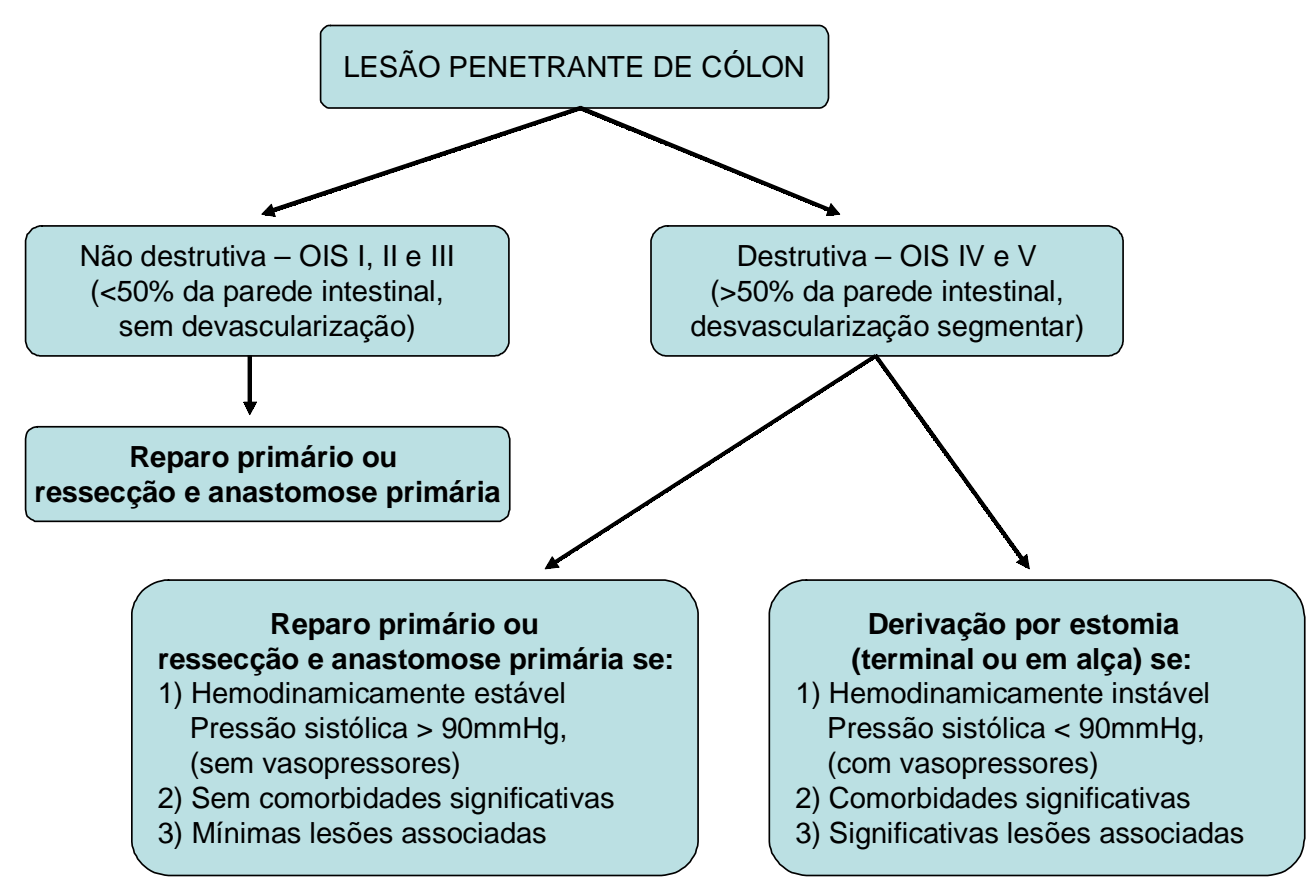

Figura 1: Algoritmo do tratamento das lesões colônicas, segundo Cleary et al. ${ }^{13}$ 


\section{6- CONCLUSÃO}

As lesões de vísceras ocas são freqüentes nos traumas abdominais, algumas vezes de difícil diagnóstico e se destacam por sua alta morbidade e mortalidade. O exame físico continua a ser o mais importante método para o diagnóstico das lesões abdominais que necessitam de cirurgia de urgência e atualmente não há exame subsidiário que, isoladamente, faça o diagnóstico conclusivo da lesão de víscera oca. O emprego de procedimentos laparoscópicos, apesar de utilizado em alguns casos, ainda necessita de estudos mais aprofundados para formalizar a indicação rotineira. Frente a suspeita de lesão de víscera oca, investigações insistentes devem ser realizadas a fim de se chegar ao diagnóstico precocemente, uma vez que resultados catastróficos podem advir do retardo no tratamento.

Motta DC, Scarpelini S. Injuries to the hollow viscera on trauma. Medicina (Ribeirão Preto) $2007 ; 40$ (4): 531-7, oct./dec.

ABSTRACT: In abdominal blunt or penetrating trauma patients, despite of the initial assessment and stabilization, careful diagnosis of associated injuries and immediate treatment in obvious cases, we must be concerned about missing injuries of hollow viscera that can disclose itself later, delaying definitive treatment, with consequent worse prognosis. Based in high suspicion related to the mechanism of trauma and associated injuries in the initial evaluation, diagnostic peritoneal lavage and radiological exams must be indicated in the effort to confirm the existence of hollow visceral injuries. Currently, there is no method with such specificity and sensitivity to define diagnosis by itself. Among the organs injured in abdominal trauma, the small bowel is the most frequent in penetrating wounds, followed by colon, stomach and duodenum. The hollow viscera injuries are less common in blunt trauma, however usually associated to higher severity and other injuries. The treatment is surgical, usually with primary repair, guided by the anatomical injury severity and patient general conditions.

Keywords: Abdominal Injuries. Viscera; injuries. Stomach; injuries. Intestine, Small; injuries. Colon; injuries.

\section{REFERÊNCIAS}

1 - Moore EE, Cogbill TH, Malangoni MA, Jurkovich GJ, Champion HR, Gennarelli TA, et al. Organ injury scaling II. Pancreas, duodenum, small bowell, colon and rectum. J Trauma1990; 30(11):1427-9

2 - Mattox KL, Feliciano DV, Moore EE. Trauma. New York: McGraw-Hill; 1999.

3 - Committee on Trauma. Advanced Trauma Life Support Student Course Manual. Chicago: American College of Surgeons; 2004.

4 - Rotondo MF, Zonies DH. The damage control sequence and underlying logic. Surg Clin North Am 1997;77:761-77.

5 - Germanos S, Gourgiotis S, Villias C, Bertucci M, Dimopoulos N, Salemis N. Damage control surgery in the abdomen: An approach for the management of severe injured patients. Int $\mathrm{J}$ Surg. 2007. [Epub ahead of print].
6 - Joshua WS, Stephan WA, Soto JA. Blunt abdominal trauma: current imaging techniques and CT findings in patients with solid organ, bowel, and mesenteric injury. Semin Ultrasound CTMRI 2007; 28(2):115-29.

7 - Sauerland S, Agresta F, Bergamaschi R, Borzellino G, Budzinski A, Champault G, et al. Laparoscopy for abdominal emergencies: evidence-based guideline of the European Association for Endoscopic Surgery. Surg. Endosc 2006; 20(1):14-29.

8 - Tejerina Alvarez EE, Holanda MS, Lopez-Espadas F, Dominguez MJ, Ots E, Diaz-Reganon J. Gastric rupture fron blunt abdomimal trauma. Injury 2004; 35(3): 228-31.

9 - Edelman DA, Wite MT, Tyburski JG; Wilson RF. Factors affecting prognosis in patients with gastric trauma. Am Surg 2007; 73(1):48-53.

10 - Vidmar D, Pleskovic A, Tonin M. Diagnosis of bowel injuries fron blunt abdominal trauma. Eur JTrauma 2003; 29(4): 220-7. 
11 - Munshi IA, DiRocco JD, Khachi G. Isolated jejunal perforation after blunt thoracoabdominal trauma. J Emerg Med 2006; $30(4): 393-5$

12 - ZhengYX, Chen L, Tao SF, Song P, Xu SM. Diagnosis and management of colonic injuries following blunt trauma. World J Gastroenterol 2007; 13(4):633-6.

13 - Cleary RK, Pomerantz RA, Lampman RM. Colon and rectal injuries. Dis Colon Rectum 2006; 49(8):1203-22.

14 - Nelson R, Singer M. Primary repair for penetrating colon injuries.Cochrane Database Syst Rev; 2007(1). Review.
15 - Frederes SA, Camargo CFG. Trauma penetrante de colon: Manejo embasado em evidências. Rev Bras Coloproctol 2002; 22 (4): 284-9.

16 - Herr MW, Gagliano RA. Historical perspective and current management of colonic and intraperitoneal rectal trauma. Courr Surg 2005; 62 (2):187-92.

Recebido em 26/06/2007

Aprovado em 27/09/2007 